\title{
The Effect of Nitric Oxide on the Rat Diaphragm at Different Frequencies of Indirect Electrical Stimulation
}

\author{
Rasha Eldeeb ${ }^{1 *}$, Hemmat Kholousy $^{2}$, Samah El-Attar ${ }^{2}$ \\ 1Physiology Department, Dubai Medical College for Girls (DMCG), Dubai, UAE \\ 2Physiology Department, College of Medicine, Cairo University, Egypt
}

\begin{abstract}
:
Introduction: Nitric oxide (NO) is a second messenger that regulates neurotransmitter release at the neuromuscular junction (nmj). Evidences accumulate on its potency as a neuro-modulator in CNS, PNS. This work investigates the effect of $N O$ on (nmj) by adding L-arginine known as NO donor.

Material and Methods: 30 albino rats' diaphragms divided into two groups. Both exposed to L-argnine followed by Bovine $\mathrm{Hb}$ and stimulated by indirect electrical stimulation. Gp 1 stimulated at $0.5 \mathrm{~Hz}$. G $\mathrm{p}_{2}$ stimulated at $100 \mathrm{~Hz}$. The amplitude of maximum contraction $(A M C)(\triangle Y)$, contraction time $(\triangle X)$ and half relaxation time $(1 / 2 R t)$ measured.
\end{abstract}

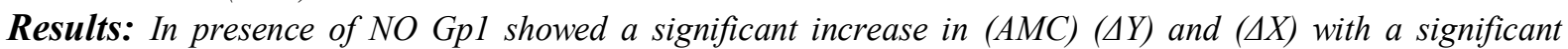
decrease in $(1 / 2 R t)$. Gp2 showed a significant increase in $(A M C)(\triangle Y)$.

Conclusion: Nitric oxide (NO) facilitates neuromuscular transmission at presynaptic level. Bovine Hemoglobin reduces Nitric oxide's induced effects.

Key words: Hemoglobin, L-arginine, nitric oxide (NO), rat diaphragm, neuromuscular transmission.

\section{Introduction}

Over the last two decades, the image of nitric oxide $(\mathrm{NO})$ has been totally changed as it was found to exhibits diverse vital roles in the brain, arteries, immune system, liver, pancreas, uterus, peripheral nerves, lung and almost every system in the human body. It is now clear that NO participate in the control of vascular tone as an antagonist of the adrenergic regulatory system. It causes smooth muscle relaxation at the vascular wall, gastrointestinal tract and it functions in both the central and peripheral nervous system ${ }^{1,2}$. Intracellular NO production is catalyzed by several isoforms of an enzyme termed, nitric oxide synthase (NOS). Neural NOsynthase is present in the sarcolemmma of type II skeletal muscle fibers ${ }^{3}$. In rats, the nitric oxide (NO) synthase pathway is present in skeletal muscle, vascular smooth muscle and motor nerve terminal ${ }^{3,4}$.

This study aims to investigate the effect of NO in rat neuromuscular preparation (diaphragm) at different frequencies of nerve stimulation and modulation of its effect by hemoglobin as NO scavenger.

\section{Materials and Methods}

The Experimental Research Committee of the Physiology Department and the ethics committee of College of Medicine, Cairo University approved all procedures. Male rats were supplied by Animal Care facility of Cairo University. The study was conducted in accordance with World Helsinki declaration.

\section{II-I Experimental protocol:}

The phrenic nerve and the diaphragm muscle of 30 Wistar rats weighing from 100-120 g each, were isolated by the method of Bulbring ${ }^{5}$. Each muscle was immersed in a 20-ml chamber containing Krebs buffer $\left(188 \mathrm{mMNaCl}, 4.7 \mathrm{mMKCl}, 1.9 \mathrm{mM} \mathrm{CaCl}, 1.2 \mathrm{mM} \mathrm{MgSO}_{4}, 25 \mathrm{mMNaHCO}_{3}, 1.2 \mathrm{mM} \mathrm{KH}_{2} \mathrm{PO}_{4}\right.$, and $11 \mathrm{mM}$ glucose) at $37^{\circ} \mathrm{C}$ and continuously aerated with a mixture of oxygen (95\%) and carbon dioxide (5\%). Isometric force was measured with isometric force transducer from which the signals was amplified and displayed simultaneously on Intracept TSC 28611 computer using analysis software phys 4 (Intracel Company).

$\mathrm{Gp}_{1}$ (no: 15) was stimulated by indirect electrical supramaximal stimuli of $0.5 \mathrm{msec}$ duration at a frequency of $0.5 \mathrm{~Hz}$. In $\mathrm{Gp}_{2}$ (no: 15 ) was stimulated by indirect electrical stimuli of $100 \mathrm{~Hz}$ frequency. In both groups a square-wave stimulator palmer electronic, England, was used to induce simple muscle twitch and the following parameters were measured ; maximal twitch force $(\Delta \mathrm{Y})$, Contraction time $(\Delta \mathrm{X})$, Half relaxation time $(1 / 2 \mathrm{RT})$

The recordings of both $\mathrm{Gp}_{1}$ and $\mathrm{Gp}_{2}$ were done three times with 10 minutes rest in between each recording, the first recording was done in presence of Krebs solution in $50 \mathrm{ml}$ bath then to study the effect of $\mathrm{NO}, 4.7 \mathrm{mM}$ of L- arginine as a NO donor was added to Krebs solution, after a contact time of 3 minutes the second recording was taken. In order to prove that the observed responses were NO mediated, $50 \mathrm{nM}$ of bovine 
$\mathrm{HB}$ was added to the $50 \mathrm{ml}$ bath containing L-arginine, after a contact time of 3 minutes the third recording was taken.

\section{II-II Drugs Used:}

L-arginine of a molecular weight of 178 and bovine hemoglobin of a molecular weight of 64.5 were purchased from MEDICO Company.

\section{II-III Statistical analysis}

Results are expressed as mean values and standard deviation. The Student's t- test was used to compare the two groups. Significance was set at the $\mathrm{P}<0.05$ level.

\section{Results}

III-I Effect of indirect supramaximal stimuli of $0.5 \mathrm{msec}$ duration and $0.5 \mathrm{~Hz}$ frequency on the rat diaphragm twitches $\left(G_{1}\right)$ : Table (1)

In presence of Krebs solution alone $\Delta \mathrm{Y}$ had a mean value of $5.85 \pm 1.75 \mathrm{~g} / \mathrm{cm}^{2}$; following the addition of L-arginine $\Delta \mathrm{Y}$ had a mean value of $8.37 \pm 1.95 \mathrm{~g} / \mathrm{cm}^{2}$ showing a significant improvement by $43.17 \%$ compared to its value in Krebs solution. After adding bovine $\mathrm{Hb}$ to the L-arginine bath $\Delta \mathrm{Y}$ had a mean value of $5.85 \pm$ $1.75 \mathrm{~g} / \mathrm{cm}^{2}$ showing a significant decrease by $30.15 \%$ compared with its corresponding mean value in the Larginine bath. Furthermore, $\Delta \mathrm{Y}$ returned back to its original mean value recorded in Krebs solution.

In the presence of Krebs solution alone $\Delta X$ had a mean value of $0.0496 \pm 0.0167 \mathrm{~g} / \mathrm{cm}^{2}$ which increased by adding L-arginine to Krebs solution to $0.0701 \pm 0.0271 \mathrm{~g} / \mathrm{cm}^{2}$ showing a significant increase by $41.41 \%$. When adding bovine $\mathrm{Hb}$ to the L-arginine bath, $\Delta \mathrm{X}$ had a mean value of $0.0452 \pm 0.0167 \mathrm{~g} / \mathrm{cm}^{2}$ showing a significant decrease by $35.55 \%$ compared with that recorded in the presence of L-arginine, although the mean value of the $\Delta X 0.0452 \pm 0.0167 \mathrm{~g} / \mathrm{cm}^{2}$ in presence of Bovine $\mathrm{HB}$ was even lower than its corresponding value $0.0496 \pm 0.0167 \mathrm{~g} / \mathrm{cm}^{2}$ in Krebs solution yet this difference was insignificant.

$1 / 2$ Rt recorded in presence of Krebs solution only had a mean value of $0.0754 \pm 0.0454 \mathrm{msec}$. which shortened significantly by $32.28 \%$ when L-arginine was added to the Krebs solution bath as it had a mean value of $0.0510 \pm 0.0237 \mathrm{msec}$.. After the addition of bovine $\mathrm{Hb}$ to $\mathrm{L}$-arginine bath, $1 / 2 \mathrm{Rt}$ had a mean value of $0.0756 \pm 0.02904 \mathrm{msec}$. increasing significantly by $48.06 \%$ compared with its value in L-arginine bath and no significant difference was observed when compared with its mean value in the presence of Krebs solution alone.

III-II Effect of indirect high frequency stimulation $(100 \mathrm{~Hz})$ on rat diaphragm twitches $\left(\mathrm{Gp}_{2}\right)$ : Table (2)

$\Delta \mathrm{Y}$ recorded in the presence of Krebs solution alone had a mean value of $14.65 \pm 2.47 \mathrm{~g} / \mathrm{cm}^{2}$, with the addition of L-arginine to the bath $\Delta \mathrm{Y}$ increased to $17.69 \pm 2.311 \mathrm{~g} / \mathrm{cm}^{2}$ showing a significant increase by $20.75 \%$ compared with its mean value recorded in presence of Krebs solution alone. Following the addition of Bovine $\mathrm{Hb}$ to the bath containing L-arginine, $\Delta \mathrm{Y}$ became $14.51 \pm 2.472 \mathrm{~g} / \mathrm{cm}^{2}$ which decreased significantly by $17.97 \%$ compared with its value in the presence of L-arginine alone. This value was insignificant when compared with its corresponding value recorded in Krebs solution.

Table (1): The effects of NO on $\left(\mathrm{Gp}_{1}\right)$; low frequency stimuli $0.5 \mathrm{~Hz}$ of indirect electrical stimulation.

\begin{tabular}{|c|c|c|c|}
\hline \multicolumn{4}{|c|}{ Changes in $\left(\mathrm{Gp}_{1}\right)$ stimulated at low frequency $0.5 \mathrm{~Hz}$ of indirect electrical stimulation } \\
\hline & Krebs solution & Krebs + L-argnine & L-argnine/Bovine $\mathrm{Hb}$ \\
\hline$\Delta \mathrm{Y}$ in $\mathbf{g} / \mathbf{c m}^{2}($ mean $\pm S D)$ & $5.85 \pm 1.75$ & $8.37 \pm 1.95$ & $5.85 \pm 1.75$ \\
\hline$\%$ of changes & & $+43.1 \%$ & $-30.1 \%$ \\
\hline $\mathbf{P}$ & & $<0.05^{*}$ & $\begin{array}{l}<0.05^{* *} \\
>0.05 \#\end{array}$ \\
\hline$\Delta \mathrm{X}$ in $\mathbf{g} / \mathbf{c m}^{2}($ mean $\pm \mathrm{SD})$ & $0.0496 \pm 0.0167$ & $0.0701 \pm 0.0217$ & $0.0452 \pm 0.0167$ \\
\hline \% of changes & & $+41.4 \%$ & $-35.5 \%$ \\
\hline $\mathbf{P}$ & & $<0.05^{*}$ & $\begin{array}{l}<0 / 05^{* *} \\
>0.05 \#\end{array}$ \\
\hline $1 / 2$ Rt in msec. $($ mean \pm SD $)$ & $0.07543 \pm 0.0454$ & $0.05106 \pm 0.0237$ & $0.0756 \pm 0.029$ \\
\hline \% of changes & & $-32.28 \%$ & $+48.06 \%$ \\
\hline $\mathbf{P}$ & & $<0.05^{*}$ & $\begin{array}{l}<0.05^{* *} \\
>0.05 \#\end{array}$ \\
\hline
\end{tabular}

* Significant value compared with its value in the presence of $L$-arginine in the bath. ** Significant value compared with its value in the presence of L-arginine/Bovine Hb in the bath. \# Insignificant value compared with its value in Krebs solution. 
Table (2): The effects of NO on (Gp $\left.\mathrm{G}_{2}\right)$; High frequency stimuli $100 \mathrm{~Hz}$ of indirect electrical stimulation.

\begin{tabular}{|l|l|l|l|}
\hline \multicolumn{4}{|c|}{ Changes in (Gp2)at high frequency 100Hz of indirect electrical stimulation } \\
\hline & Krebs solution & $\begin{array}{l}\text { Krebs + L- } \\
\text { argnine }\end{array}$ & L-argnine/Bovine Hb \\
\hline $\boldsymbol{\Delta}$ Y in $\mathbf{~ g / \mathbf { c m } ^ { 2 }}($ mean $\pm \mathrm{SD})$ & $14.650 \pm 2.47$ & $17.693 \pm 2.311$ & $14.513 \pm 2.472$ \\
\hline \% of changes & & $+20.7 \%$ & $-17.97 \%$ \\
\hline P & & $<0.05^{*}$ & $\begin{array}{l}<0.05^{* *} \\
>0.05 \#\end{array}$ \\
\hline
\end{tabular}

\section{* Significant value compared with its value in the presence of $\mathbf{L}$-arginine in the bath. ** Significant value compared with its value in the presence of $\mathrm{L}$-arginine/Bovine $\mathrm{Hb}$ in the bath. \# Insignificant value compared with its value in Kreb's solution.}

\section{Discussion}

Nitric oxide (NO) is an important multifunctional second messenger with numerous biological functions it contributes to the regulation of several processes such as excitation contraction coupling and mitochondrial respiration. $\mathrm{NO}$ is an important modulator of synaptic transmission at the neuromuscular junction (NMJ), as exogenously applied NO reduces neurotransmitter release in immature and mature frog NMJS ${ }^{6,7}, \mathrm{NO}$ reduces the sensitivity to neurotransmitters of perisynaptic Schwann cells (PSCs), glial cells at the frog NMJ ${ }^{8}$ and NOSs are found in skeletal muscle fibers where they are concentrated at the muscle end plate ${ }^{9}$.

In the rat, NO is synthesized by NO synthase that is present in the sarcolemma of type II fibers of skeletal muscle, vascular smooth and motor nerve terminals ${ }^{10}$.NOS is a stereospecific enzyme, so the effects induced by L-arginine are not observed in the presence of $\mathrm{D}$-arginine under similar experimental conditions ${ }^{11,12}$. Several analogues of L-arginine (NG-monomethylL-arginine; NG-nitro-L-arginine (L-NOARG); L-arginine methyl ester; L-arginine ethyl ester, and NG-nitro-L-arginine methyl ester) are used as inhibitors of NOS. The effects produced by endogenous NO (from L-arginine) are pharmacologically similar to those induced by NO released from an exogenous source such as 3-(4-morpholinyl)-syndononeimine (SIN-1) or S-nitroso-Nacetylpenicillamine(SNAP) ${ }^{13}$.

The present study was designed to focus the light on the effect of NO on the contractility of rat diaphragm using indirect electrical stimulation at low $(0.5 \mathrm{~Hz})$ and high $(100 \mathrm{~Hz})$ frequencies.

Indirect electrical stimulation of the rat diaphragm preparation at $0.5 \mathrm{~Hz}$ in presence of $\mathrm{L}$-arginine $(4.7$ $\mathrm{mM})$ as a precursor of NO ${ }^{13,14,15}$ showed a significant increase in the amplitude of muscular contraction $(\Delta \mathrm{Y})$ and contraction time $(\Delta \mathrm{X})$ by $43.17 \%$ and $41.41 \%$ respectively with a significant decrease in the relaxation time $(1 / 2 \mathrm{Rt})$ by $32.28 \%$. Results were in agreement with Ambiel and ALves-Do-Prado who found that NO precursor L-argnine (4.7-9.4mM) acting at the pre-synaptic level produced a dose - dependent increase of AMC in rat neuromuscular preparations when stimulated indirectly at $0.2 \mathrm{~Hz}$. However, Queiroz and Alves-Do-Prado, reported that the amplitude of muscle contraction $(\Delta \mathrm{Y})$ was stable when the rat diaphragm was indirectly stimulated at $0.2 \mathrm{~Hz}$. A progressive increase in $\mathrm{AMC}$ was observed at 5 and $50 \mathrm{~Hz}$ respectively. In this regard, it is interesting to note that nNOS and c.GMP - dependent protien kinase, which are the primary effectors of NO, were found to be concentrated at the neuromuscular end plate. Moreover, Wang et al. in 1995 noticed that c.GMP-dependent kinase phosphorylate the nicotinic acetylcholine receptor subunits in cultured myocytes. ${ }^{16}$, 17,18 .

Studies found that NO acting at presynaptic level might increase acetylcholine release from motor nerve terminals. It could be modulated by endogenous agents such as adenosine, catecholamine, metabolic arachidonic acid, calcitonin gene - related peptide, substance P, VIP and hormones, thus NO might possibly represent another modulating factor of acetylcholine release by the motor nerve terminal ${ }^{13,14,15}$.

UC et al., 1999 suggested that activation of a soluble guanylatecyclase leading to the production of $\mathrm{c}$. GMP seems to be the main mechanism by which NOmodulates transmitter release during application of low frequency electrical stimulation. $\mathrm{NO}$ also, could induce post-translational changesof synaptic proteins involved in the regulation of the exocytosis-endocytosiscycle. In addition, there was evidence that NO facilitated N-type $\mathrm{Ca}^{2+}$ channel activation via a c.GMP-PKG pathway. These channels are clustered at active zones of the neuromuscular junction (nmj), regulating transmitter release ${ }^{19}$.

In contrast Yong and William, 2001 found that $\mathrm{NO}$ act as an inhibitory chemical transmitter at the neuromuscular junction. They observed the large increase in muscle volume during development required an expansion of presynaptic terminal, this matching in size could only be accomplished through retrograde signals, as the neuromuscular junction was fully differentiated at the end of embryogenesis. The immunocytochemical staining c.GMP in the presynaptic terminal, together with the physiological demonstration of an NO-induced vesicle release provided evidence that NO might serve as a retrograde signal for activity-dependent suppression at the neuromuscular synapse. Therefore, they suggested that synaptic suppression may be triggered by NO released from a postsynaptic myocyte that fire asynchronously to the presynaptic terminal ${ }^{20,21}$. 
In addition, skeletal muscle nNOS was reported to play a role in the regulation of a number of cellular processes such as contractile activity, glucose uptake, and blood flow distribution, but the importance of sarcolemmal targeting of nNOS for these regulatory functions is largely unknown ${ }^{22}$ More importantly, authors found that diaphragmatic NOS activity during postnatal development was strongly controlled at multiple levels, including gene transcription. An endogenous inhibitor, the newly discovered PIN (protein inhibitor of nNOS) is known to be widely expressedin various skeletal muscles including the diaphragm, and to inhibit nNOS activity by preventing its dimerization. PIN is localized at the sarcolemma of skeletal muscle fibers and is transcriptionally controlled during skeletal muscle development. Furthermore, recent evidence indicated the presence of caveolin-3 (a membrane-boundprotein) in normal skeletal muscles that interacts selectively with nNOS and potently inhibitsits activity). Finally, it has been well established that endogenous NO synthesis in a variety of cells, including skeletal muscles, attenuates mitochondrial respiration by reversibly inhibiting mitochondrial enzymes such as cytochrome-c oxidase ${ }^{23,24}$.

Whether nitric oxide (NO) facilitated or impaired the neuromuscular transmission in diaphragm preparations indirectly stimulated at low frequency, we suggested that it was depending on the followed experimental protocol. Also, it was likely that the release of endogenous NO differs with NO donors used in terms of concentration and duration. In combination, these data suggested that exposures to NO have complex and multi-factorial effects on contractile function.

Since rat diaphragm was demonstrated to be anatomically formed of $\sim 26 \%$ type I, $23 \%$ type IIa and $52 \%$ type IIx/b fibers and studies demonstrated that the direction of the change in the force in response to NO donors depends significantly on the interval between contractions. When muscles are stimulated more frequently, force increases in response to NO; the opposite occurs when the stimulation interval increases ${ }^{25}$ and at $100-\mathrm{Hz}$ stimulations, all fibers would contract synchronously to isometric contraction, reaching almost full tetany and sharing in the net isometric force development ${ }^{4}$.Thus, this work aimed to determine whether NO was involved in the high-frequency-induced force changes at the neuromuscular junction, in a trial to assess the previously recorded findings.

In this study indirect electrical stimulation of the rat diaphragm at $100 \mathrm{~Hz}$, tetanic contractions of the muscle developed and recorded. The amplitude of maximum contraction $(\Delta \mathrm{Y})$ was observed to be improved by $20.75 \%$ after the addition of L-arginine to the rat diaphragm preparation. The recorded results were in agreement with Schuman and Madison who suggested that, during high-frequency stimulation, endogenous and exogenous NO increase basal acetylcholine release from central and peripheral cholinergic neurons. It had been proposed that acetylcholine, in addition to its action on subsynaptic membranes, also acts on prejunctional cholinoceptors to change the acetylcholine mobilization process and thus to control the neurotransmitter output during tetanic stimulation ${ }^{26}$.

In addition, Queiroz and Alves -Do-Prado 2001 suggested that, the increase in the amplitude of muscle contraction produced at different frequencies, depend on the metabolism of L-argnine producing additional NO in the tissues and its ability to scavenge superoxide anions. They reported that NOS activity might change according to the applied frequency of stimulation to the motor nerve ${ }^{3}$.

It was also possible that during high-frequency stimulation, presynaptic as well as postsynaptic components are activated through a variety of signal transduction cascades. Lawler and $\mathrm{Hu}, 2000$ documented that the high-frequency-induced changes in muscle contraction, was partially modulated by NO possibly via c.GMP-independent mechanisms and that, the tonic production of NO would originate from the muscle fibers to serve as a feedback signal for synapse maintenance. NO might increase the release of acetyl choline from motor nerve terminals, with a consequent activation of inhibitory presynaptic muscarinic receptors. Furthermore, Silvagno et al., 1996 reported that, the increased NO production would also reduce the sensitivity of PSCs to neurotransmitters as it reduced the $\mathrm{Ca}^{2+}$ responses elicited in PSCs by transmitter substances ${ }^{27,28}$.

In this study in order to confirm that the obtained results were NO mediated, bovine hemoglobin - 50 $\mathrm{nM}$, known to be NO scavenger -was added to the bath $(50 \mathrm{ml})$ containing L-argnine. Indirect stimulation of rat diaphragm at frequencies of $0.5 \mathrm{~Hz}$ and $100 \mathrm{~Hz}$ showed a decrease in both $\Delta \mathrm{Y}$ of simple muscle twitch and the tetanic contraction by $30.15 \%$ and $17.97 \%$ respectively . In addition, there was no significant change in the mean value of $\Delta \mathrm{Y}$ at low frequency $(0.5 \mathrm{~Hz})$ in presence of bovine hemoglobin compared to its initial mean values in kreb's solution alone. Therefore, depending on these data, we can accept that the observed results were, to a great extent, due to $\mathrm{NO}$ enriched medium.

Finally we can conclude that NO formed from L-arginine through the NO-synthase pathway present in the sarcolemma of type II fibers of rat skeletal muscle enhances the contractility of the rat diaphragm when the phrenic-nerve diaphragm preparation is indirectly stimulated. Therefore, we can propose that NO increases the amplitude of muscular contraction when it interacts at the presynaptic level. Whether the presynaptic action of NO will be altered by its postsynaptic action or not, further investigation and comparison while applying a direct electrical stimulation at the neuromuscular junction $(\mathrm{nmj})$ is recommended. 


\section{Conclusion}

This study concludes that nitric oxide (NO) facilitates the synaptic transmission of the neuromuscular junction at the presynaptic level and that hemoglobin acts as nitric oxide (NO) scavenger that antagonizes or reduces its effect.

\section{References:}

[1]. Zhu X, Heunks LM, Ennen L, Machiels HA, Van Der Heijden HF, Dekhuijzen PN .Nitric oxide modulates neuromuscular transmission during hypoxia in rat diaphragm. Muscle Nerve 2006; 33(1):104-12.

[2]. Mukhtarov MR, UrazaevAKh, Nikol'skiı̌ EE, Vyskocil F. Modulation of the intensity of the non-quantal transmitter release by nitric oxide (NO) at the neuromuscular junction.RossFiziolZhIm I M Sechenova 2000; 86(3):335-42.

[3]. Queiroz R.N and Alves - Do-Prado .Effect of L-argnine on the diaphragm muscle twitches elicited at different frequencies of nerve stimulation. Braz.J. Med. Res 2001; 34(6): 825-828.

[4]. Kobzik L., Reid M.B., Bredt D.S. and Stamler J.S. Nitric oxide in skeletal muscle.Nature 1994.372:546-548.

[5]. Bulbring E. Observation on the isolated phrenic nerve diaphragm preparation of the rat. Br J PharmacChemother 1946: 38.

[6]. Wang T, Xie Z, Lu B Nitric oxide mediates activity - dependent synaptic suppression at developing neuromuscular synapses. Nature 1995; 374: 262-266,

[7]. Lindgren C.A.and Laird M.V .Nitroprusside inhibits neurotransmitter release at the frog neuromuscular junction. Neuro report 1994; 5:2205-2208.

[8]. Balon T.W. and Nadler J. L. Nitric oxide release is present from incubated skeletal muscle preparations. J ApplPhysiol 1994;77: 2519-2521.

[9]. Imran N. MungrueandDavid S. Bredt :nNOS at a glance:implications for brainandbrawnJournal of Cell Science 2004;117:26272629

[10]. Ribera J, Marsal J, Casanovas A, Hukkanen M, Tarabal O \&Esquerda JE Nitric oxide synthase in rat neuromuscular junctions and in nerve terminals of Torpedo electric organ: its role as regulator of acetylcholine release. Journal of Neuroscience Research 1998; 51: $90-102$.

[11]. Moritoki H, Ueda H, Yamamoto T, Hisayama T \& Takeuchi S. L-Arginine induces relaxation of rat aorta possibly through nonendothelial nitric oxide formation. British Journal of Pharmacology1991; 102: 841-846.

[12]. Sakuma I, Stueher DJ, Gross SS, Nathen C \& Levi R. Identification of arginine as a precursor of endothelium-derived relaxing factor. Proceedings of the National Academy of Sciences, USA 1988; 85: 8664-8667.

[13]. Moncada S, Higgs A \&Furchgott R XIV. International union of pharmacology nomenclature in nitric oxide research.Pharmacological Reviews1997; 49: 137-142.

[14]. Xiaoping Zhu, Leo M. A. Heunks, Herwin A. Machiels, Leo Ennen and P. N. Richard Dekhuijzen.Effects of modulation of nitric oxide on rat diaphragm isotonic contractility during hypoxia. J ApplPhysiol 2003; 94:612-620.

[15]. Fujii Y.,Guo Y. and Hussain S.N.A . Regulation of nitric oxide production in response to skeletal muscle activation. J appl. Physiol 1998; 85( 6): 2330-2336.

[16]. Ambiel C.R and Alves -Do Prado W. Antagonism by hemoglobin of effects induced by L-argnine in neuromuscular preparations from rats. Braz. J. Med. Biol. Res 2001; 34(4): 549-552.

[17]. Queiroz R.N and Alves -Do-Prado .Effect of L-argnine on the diaphragm muscle twitches elicited at different frequencies of nerve stimulation. Braz.J. Med. Res 2001; 34(6): 825-828.

[18]. Wang T. Xie Z., and LueB.Nitric oxide mediates activity-dependent synaptic suppression at developing neuromuscular synapses. Nature 1995; 374:262-266.

[19]. Uc A. Oh S.T. Murray J. A. Clark E. and Conklin J.L Biphasic relaxation of the oesophageal sphincter: role of NO, VIP and CGRP. American J Physiol 1999; 277, G548-554

[20]. Yong Z. and William G.P. Nitric oxide contracts longitudinal smooth muscle of opossum oesophagus via excitation - contraction coupling. J of physiology 2001; 536(1): 133-140.

[21]. Olgart C., Wiklund N.P. and Gustafsson L.E. Blockade of nitric oxide - evoked contractions by an inhibitor of guanylylcyclase.NeuroReport 1997;8 (15): 3355-3358.

[22]. Mukhtarvo M.R., Urazaev A.K., Nikolsky E.E and VyskocilF.Effect of nitric oxide and NO synthase inhibition on nonquantal acetylcholine release in the rat diaphragm. Eur J Neurosci.2000; 12: 980-986.

[23]. Jaffrey S.R. and Snyder S.H. PIN: an associated protein inhibitor of neuronal nitric oxide synthase. Science1996; $274: 774-777$.

[24]. Guo Y., Greenwood T., Petrof J. and Hussain S.N.A .Expression and regulation of protein inhibitor of neural nitric oxide synthase in ventilatory muscles. Am. J. Cell and Mol. Biol 1999; 20( 2): 319-326.

[25]. Morrison R.J., Miller C.C., and Reid M.B.Nitric oxide effects on shorting velocity and power production in the rat diaphragm. J ApplPhysiol1996; 80: 1065-1069.

[26]. Schuman E.M. and Madison D.V. Nitric oxide and synaptic function. Annu. Rev. Neurosci 1994; $17,153-183$.

[27]. Lawler J.M. and $\mathrm{Hu} \mathrm{Z}$. Interaction of nitric oxide and reactive oxygen species on rat diaphragm contractility.ActaPhysiolScand 2000; 169: 229-236.

[28]. Silvagno F. Xia H. and Bredt D.S. Neuronal nitric oxide synthase $-U$, an alternative spliced isoform expressed in differentiated skeletal muscle. J BiolChem1996; 271: 11204-11208. 